\title{
Exact Euclidean Medial Axis in Higher Resolution
}

\author{
André Vital Saúde ${ }^{1,2, \star}$, Michel Couprie ${ }^{2}$, and Roberto Lotufo ${ }^{1}$ \\ ${ }^{1}$ State University of Campinas, School of Electrical and Computer Engineering, \\ DCA-FEEC-UNICAMP, Caixa Postal 6101, 13081-970 Campinas/SP, Brazil \\ \{andrevit, lotufo\}@dca.fee.unicamp.br \\ ${ }^{2}$ Institut Gaspard-Monge, Laboratoire A2SI, Groupe ESIEE \\ Cité Descartes, BP 99, 93162 Noisy-le-Grand Cedex, France \\ \{vitalsaa, coupriem\}@esiee.fr
}

\begin{abstract}
The notion of skeleton plays a major role in shape analysis. Some usually desirable characteristics of a skeleton are: sufficient for the reconstruction of the original object, centered, thin and homotopic. The Euclidean Medial Axis presents all these characteristics in a continuous framework. In the discrete case, the Exact Euclidean Medial Axis (MA) is also sufficient for reconstruction and centered. It no longer preserves homotopy but it can be combined with a homotopic thinning to generate homotopic skeletons. The thinness of the MA, however, may be discussed. In this paper we present the definition of the Exact Euclidean Medial Axis on Higher Resolution which has the same properties as the MA but with a better thinness characteristic, against the price of rising resolution. We provide an efficient algorithm to compute it.
\end{abstract}

\section{Introduction}

In 1961, Blum [1 introduced the notion of medial axis or skeleton, which has since been the subject of numerous theoretical studies and has also proved its usefulness in practical applications. Consider a subset X (called object) of a metric space. The medial axis of $\mathrm{X}$ is the set composed by the centers of the maximal balls for $\mathrm{X}$, that is, the balls which are included in $\mathrm{X}$ but which are not included in any other ball included in X.

Although originally defined in the continuous plane, the medial axis can be defined using the same terms in the $n$-dimensional discrete grid $\mathbb{Z}^{n}$. The discrete medial axis is a set of points which is, by nature, centered in the object with respect to the distance which is used to define the notion of ball. To achieve a certain degree of rotation invariance, the Euclidean distance between points of $\mathbb{Z}^{n}$ may be used. Nevertheless even in this case, the medial axis has not, in general, the same nice properties as its continuous counterpart. In particular, the properties of thinness and homotopy are lost (see for example Figure 1(a), where the medial axis is "two-pixels thick" in some places, and has not the same number of connected components as the original object).

* A.V. Saúde is thankful to the financial support from Fapesp and Capes, Brazil.

A. Kuba, L.G. Nyúl, and K. Palágyi (Eds.): DGCI 2006, LNCS 4245, pp. 605616 2006.

(C) Springer-Verlag Berlin Heidelberg 2006 
Topological aspects are out of the scope of this paper. Nevertheless, let us mention that in order to obtain an homotopic skeleton which contains the medial axis, the use of guided and constrained discrete homotopic transformations has been proposed by several authors (see e.g. [2, 3, 4]).

On the other hand, the problem of thickness of the skeleton has been tackled in different ways. Some authors use an asymmetric thinning step in order to reduce two-pixel thick configurations. But in this case, the result cannot be considered anymore as centered with respect to the original object. Furthermore the property of reconstruction is lost. In order to get a thin medial axis while preserving centeredness (in the sense of the so-called 8-distance), G. Bertrand introduced the notion of derived grid [5]. Also, in the study of discrete topology-preserving transformations, several recent works promote the use of the Khalimsky grid or its variations [6, 7, 8, 9, 10. From a geometrical point of view, the Khalimsky grid $\mathbb{H}^{n}$ associated to $\mathbb{Z}^{n}$ can be embedded in $\left[\frac{1}{2} \mathbb{Z}\right]^{n}$, that is, by doubling the resolution of the grid for each dimension. Starting from an initial object in $\mathbb{Z}^{n}$, a "model" of this object in $\mathbb{H}^{n}$ can be computed and then thinned by a symmetrical algorithm [9], producing a result which is both centered and thin.

The drawback of the above approaches lies in their sensitivity to rotations. Since they implicitly or explicitly rely on the 4- or 8-distance, the skeletons can be quite different for an object and its rotation by, say, $\pi / 4$. Our aim in this paper is to provide a notion of medial axis in a doubled resolution grid, based on the Euclidean distance, and an efficient algorithm to compute it. In addition to the Euclidean balls centered on points of $\mathbb{Z}^{n}$, we will also consider the balls centered on points of $\left[\frac{1}{2} \mathbb{Z}\right]^{n}$ (see Figure $\mathbb{1}(\mathrm{b})$ ).

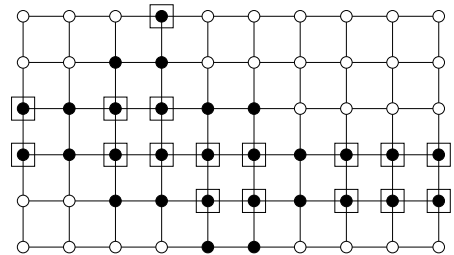

(a)

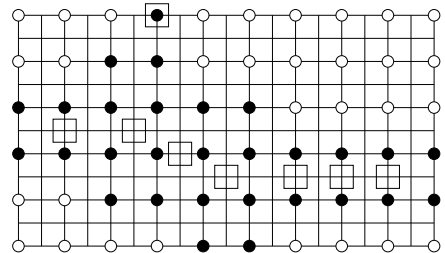

(b)

Fig. 1. (a)The object $X \subset \mathbb{Z}^{2}$ is depicted in black. The squares mark the points of the medial axis, based on the Euclidean distance; (b)The medial axis of $\mathrm{X}$ in the doubled resolution grid (squares).

Dealing with the Euclidean distance in $\mathbb{Z}^{n}$ is far from easy. Many algorithms found in the literature only compute approximations of the Euclidean distance transform (e.g. [1]) or medial axis (e.g. [12]). A simple to implement, optimal algorithm for building exact Euclidean distance maps was proposed only in 1994 [13, 14, 15, and efficient algorithms to compute exact Euclidean medial 
axes were not known before 2003 [16,17, 18. The proofs for our algorithm will be provided in an extended version of this paper; meanwhile they are available online in [19].

Let us briefly and informally sketch the proposed method. First, rather than using the grid $\left[\frac{1}{2} \mathbb{Z}\right]^{n}$, we transform the original object $\mathrm{X}$ by doubling the coordinates of all its points and consider the grid $\mathbb{Z}^{n}$ as the doubled resolution grid. This choice leads to simpler notations and proofs. Then, we consider the set $\mathrm{X}_{h}$ composed of all the points of $\mathbb{Z}^{n}$ which are in the neighborhood of a point of $\mathrm{X}$ (scaled). We compute the exact squared Euclidean distance map of $\mathrm{X}_{h}$ using a linear-time algorithm [13,14. Finally, we propose an efficient algorithm to extract the higher resolution medial axis of $\mathrm{X}$ from this distance map in $2 \mathrm{D}$ and $3 \mathrm{D}$. This algorithm is based on the same idea as the one proposed by Rémy and Thiel [16, 18 and is also based on pre-computed lookup tables, following the approach originally proposed in [20]. The biggest of these tables is indeed shared by Rémy and Thiel's method and ours. Obtaining a medial axis for an object $\mathrm{X}$ from a distance map relative to a different object $\mathrm{X}_{h}$ is not obvious, and we present several intermediate properties to establish this fact. It should be noted that the naive solution, which consists of doubling the resolution and using the classical Euclidean medial axis, does not provide a satisfactory result.

\section{Basic Notions}

We denote by $\mathbb{Z}$ the set of integers, and by $\mathbb{N}$ the set of nonnegative integers. Let $\mathrm{X} \subset \mathbb{Z}^{n}$, we denote by $\overline{\mathrm{X}}$ the complementary of $\mathrm{X}$. We denote by $(y-x)^{2}$ the squared Euclidean distance between two points $x \in \mathbb{Z}^{n}$ and $y \in \mathbb{Z}^{n}$.

Let $\mathrm{X} \subset \mathbb{Z}^{n}$, the squared Euclidean distance transform of $\mathrm{X}$, denoted by $D_{\mathrm{X}}^{2}$, associates to each point $x \in \mathrm{X}$ its squared Euclidean distance to the nearest point in $\overline{\mathrm{X}}: D_{\mathrm{X}}^{2}(x)=\min \left\{(y-x)^{2}, y \in \overline{\mathrm{X}}\right\}$.

Let $x$ be a point in $\mathbb{Z}^{n}$ and let $R$ be a positive integer. The set of points $y$ of $\mathbb{Z}^{n}$ such that $(y-x)^{2}=R$ will be of particular interest in what follows. Each such point $y$ corresponds to a decomposition of $R$ into a sum of $n$ square integers. We introduce some notations to deal with square decompositions of integers.

Definition 1 (Square Decomposition). Let $n \in \mathbb{N}, R \in \mathbb{N}$, the $n$-uple $\left(r_{1}, r_{2}, \ldots, r_{n}\right) \in \mathbb{N}^{n}$ is a square $n$-decomposition of $R$ if $r_{1} \geq r_{2} \geq \ldots \geq r_{n} \geq 0$ and $\left(r_{1}^{2}+r_{2}^{2}+\ldots+r_{n}^{2}\right)=R$. We denote by $S Q D_{n}(R)$ the set of all square $n$-decompositions of $R$.

There are other decompositions of $R$ into the sum of $n$ squares, satisfying only the second condition. They may be obtained from the above decompositions by permutations and sign changes.

We set $\mathbb{D}_{n}=\left\{R \in \mathbb{N} \mid \mathrm{SQD}_{n}(R) \neq \emptyset\right\}$, the set of $n$-decomposable integers. It is known that for any $n \geq 4, \mathbb{D}_{n}=\mathbb{N}$ (Lagrange's theorem, see [21], Section $20.5)$. 
Let $R \in \mathbb{N}$, we define:

$$
\begin{aligned}
& \bar{R}=\min \left\{\delta \in \mathbb{D}_{n} \mid \delta \geq R\right\} ; \\
& R^{+}=\overline{R+1} \\
& \underline{R}=\max \left\{\delta \in \mathbb{D}_{n} \mid \delta \leq R\right\} ; \\
& R^{-}=\underline{R-1}
\end{aligned}
$$

Let $R \in \mathbb{N}$. Observe that, if $R \in \mathbb{D}_{n}$ then $\bar{R}=\underline{R}=R$ and $\left(R^{-}\right)^{+}=\left(R^{+}\right)^{-}=$ $R$. In addition, if $R \notin \mathbb{D}_{n}$ then $\bar{R}=R^{+}$and $\underline{R}=\bar{R}^{-}$.

Definition 2 (Euclidean Ball). Let $x \in \mathbb{Z}^{n}, R \in \mathbb{N}$, we denote by $B \leq(x, R)$ the Euclidean ball centered in $x$ with (squared) radius $R$ and we denote by $B^{<}(x, R)$ the Euclidean ball centered in $x$ with (squared) strict radius $R$, where $B \leq(x, R)=\left\{y \in \mathbb{Z}^{n},(x-y)^{2} \leq R\right\}$ and $B^{<}(x, R)=\left\{y \in \mathbb{Z}^{n},(x-y)^{2}<R\right\}$.

Note that $B \leq(x, R)=B^{<}\left(x, R^{+}\right)$and $B^{<}(x, R)=B^{\leq}\left(x, R^{-}\right)$.

Definition 3 (Maximal Ball). Let $\mathrm{X} \subset \mathbb{Z}^{n}, x \in \mathrm{X}, R \in \mathbb{N}$, a) $A$ ball $B^{<}(x, R) \subseteq \mathrm{X}$ is a maximal ball for $x$ in $\mathrm{X}$ if it is the largest ball centered in $x$ and included in $\mathrm{X}$, i.e., $\forall R^{\prime} \in \mathbb{N}, B^{<}(x, R) \subseteq B^{<}\left(x, R^{\prime}\right) \subseteq \mathrm{X} \Rightarrow$ $B^{<}(x, R)=B^{<}\left(x, R^{\prime}\right)$;

b) $A$ ball $B^{<}(x, R) \subseteq \mathrm{X}$ is a maximal ball for $\mathrm{X}$ if it is not strictly included in any other ball included in $\mathrm{X}$, i.e., $\forall R^{\prime} \in \mathbb{N}, \forall y \in \mathrm{X}, B^{<}(x, R) \subseteq B^{<}\left(y, R^{\prime}\right) \subseteq$ $\mathrm{X} \Rightarrow B^{<}(x, R)=B^{<}\left(y, R^{\prime}\right)$.

Proposition 1. Let $\mathrm{X} \subset \mathbb{Z}^{n}, x \in \mathrm{X}$ and $R \in \mathbb{D}_{n}$. The ball $B^{<}(x, R)$ is maximal for $x$ in $\mathrm{X}$ if and only if $R=D_{\mathrm{X}}^{2}(x)$.

Observe that, if $B^{<}(x, R)$, with $R \in \mathbb{N}$, is maximal for $\mathrm{X}$, then it is maximal for $x$ in $\mathrm{X}$. Now let us recall the definition of the medial axis [1].

Definition 4 (Medial Axis). Let $\mathrm{X} \subset \mathbb{Z}^{n}$, the medial axis of $\mathrm{X}$, denoted by $M A(\mathrm{X})$, is the set of the centers of all the maximal balls for $\mathrm{X}$.

\section{Euclidean Medial Axis in Higher Resolution}

The goal of changing resolution is to extract a medial axis of the object $\mathrm{X}$ by considering a new family of Euclidean balls which are not necessarily centered on points of X. More precisely, we also take into account Euclidean balls centered on the vertices of a doubled resolution grid. For simplicity, instead of considering half integers for the coordinates in the higher resolution grid, we begin by doubling coordinates of the original object. Thus $\mathbb{Z}^{n}$ is used as the higher resolution grid, and the points with only even coordinates constitute the support of the scaled original image.

Definition 5. Let $i \in\{0 \ldots n\}$. We define the set $E_{i} \subset \mathbb{Z}^{n}$ as the set of elements in $\mathbb{Z}^{n}$ with exactly $i$ even coordinates, more precisely $E_{i}=\left\{\left(z_{1}, z_{2}, \ldots, z_{n}\right) \in\right.$ $\left.\mathbb{Z}^{n}, \sum_{j=1}^{n}\left(\left(z_{j}+1\right) \bmod 2\right)=i\right\}$.

Let $\mathrm{X} \subset \mathbb{Z}^{n}$, we write $E_{i}(\mathrm{X})=E_{i} \cap \mathrm{X}$. The family $\left\{E_{i}\right\}_{i=0 . . n}$ forms a partition of $\mathbb{Z}^{n}$, i.e., $\bigcup_{i=0 . . n} E_{i}=\mathbb{Z}^{n}$ and $\forall i, j \in\{0 \ldots n\}, i \neq j \Rightarrow E_{i} \cap E_{j}=\emptyset$. 
Definition 6 (Neighborhood). Let $x \in \mathbb{Z}^{n}$, we define the neighborhood of $x$ as the set $N_{n}(x)=\left\{y \in \mathbb{Z}^{n}\left|\max _{i=1} . . n\right| y_{i}-x_{i} \mid \leq 1\right\}$. Let $\mathrm{X} \subset \mathbb{Z}^{n}$, we define the set $N_{n}(\mathrm{X})=\bigcup_{z \in \mathrm{X}} N_{n}(z)$.

Definition 7. Let $\mathrm{X} \subset \mathbb{Z}^{n}$, we define $\phi(\mathrm{X})$ and $\phi^{-1}(\mathrm{X})$ by $\phi(\mathrm{X})=\{2 z, z \in \mathrm{X}\}$ and $\phi^{-1}(\mathrm{X})=\{z, 2 z \in \mathrm{X}\}$.

Definition 8 ( $H$-transform). Let $\mathrm{X} \subset \mathbb{Z}^{n}$, the $H$-transform of $\mathrm{X}$, denoted by $\mathcal{H}(\mathrm{X})$, is defined by $\mathcal{H}(\mathrm{X})=N_{n}(\phi(\mathrm{X}))$.

Figure 2 illustrates the $H$-transform of a set in $\mathbb{Z}^{2}$. Let $\mathrm{X} \subset \mathbb{Z}^{n}$, observe that $\phi(\mathrm{X}) \subseteq E_{n}$, that $\phi^{-1}(\phi(\mathrm{X}))=\mathrm{X}$ and that $E_{n}(\mathcal{H}(\mathrm{X}))=\phi(\mathrm{X})$, hence $\phi^{-1}\left(E_{n}(\mathcal{H}(\mathrm{X}))\right)=\mathrm{X}$. The following proposition is elementary.

Proposition 2. The $H$-transform is increasing, i.e., for each $A, B \subset \mathbb{Z}^{n}, A \subset$ $B \Rightarrow \mathcal{H}(A) \subset \mathcal{H}(B)$.

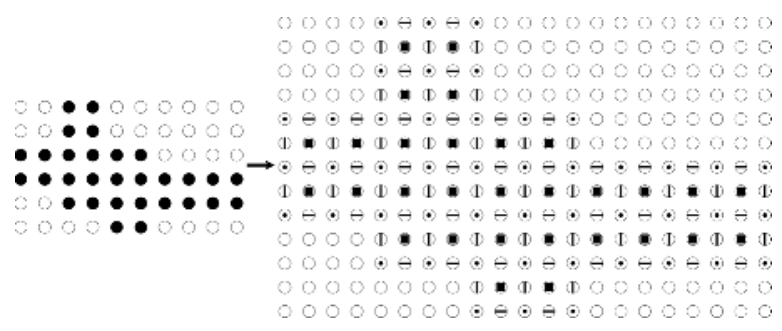

Fig. 2. $H$-transform of a set in $\mathbb{Z}^{2}$. On the left, the set $\mathrm{X}$ (in black), and on the right, the set $\mathcal{H}(\mathrm{X})$. Elements of $\mathcal{H}(\mathrm{X})$ are marked based on the sets of the partition: $E_{0}$ (with a dot), $E_{1}$ (with a vertical or horizontal line) and $E_{2}$ (with a square).

Definition 9 ( $E_{n}$-tight). Let $\mathrm{X} \subset \mathbb{Z}^{n}, x \in \mathrm{X}, R \in \mathbb{D}_{n}$. The ball $B \leq(x, R)$ is said to be $E_{n}$-tight if there is no n-decomposable integer $R^{\prime}<R$ such that $E_{n}\left(B \leq\left(x, R^{\prime}\right)\right)=E_{n}(B \leq(x, R))$.

It can easily be seen that $B \leq(x, R)$ is $E_{n}$-tight if and only if there exists $y \in$ $E_{n}(B \leq(x, R))$ such that $(x-y)^{2}=R$. The following proposition will play an important role to prove the algorithms that we are going to propose. Put briefly, it says that any $E_{n}$-tight ball $B$ is included in $N_{n}\left(E_{n}(B)\right)$.

Proposition 3. Let $n \in \mathbb{N}, n \leq 3$. Let $B=B \leq(x, R)$, where $x \in \mathbb{Z}^{n}, R \in \mathbb{D}_{n}$, be an $E_{n}$-tight ball. For any $z \in B$, there exists a point $w \in N_{n}(z)$ such that $w \in E_{n}(B)$, i.e., $B \subseteq N_{n}\left(E_{n}(B)\right)$.

Although this proposition may seem simple, it is in fact false in general but true at least in dimensions 2 and 3. Furthermore, the counter-example of Figure 3 shows that the condition " $E_{n}$-tight" is indeed necessary for the proposition. With this proposition, we will be able to justify our algorithms, and the practical applications are mostly in dimensions 2 and 3. 


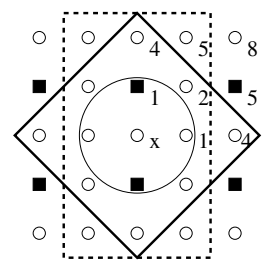

Fig. 3. Consider the ball $B=B \leq(x, 4)$, surrounded by a square. The points of $E_{n}$ are marked by black squares. We see that $B$ is not $E_{n}$-tight, and that $B$ is not included in $N_{n}\left(E_{n}(B)\right)$ (surrounded by a dashed rectangle). On the other hand, the ball $B^{\prime}=$ $B^{\leq}(x, 1)$ (surrounded by a circle) is $E_{n}$-tight and included in $N_{n}\left(E_{n}\left(B^{\prime}\right)\right)$.

\section{$\boldsymbol{E}_{\boldsymbol{n}}$-Balls and Higher Resolution Medial Axis}

For any $x \in \mathbb{Z}^{n}, R \in \mathbb{D}_{n}$, the set $E_{n}\left(B^{<}(x, R)\right)$ is called an $E_{n}$-ball, it can be seen as an Euclidean ball in $E_{n}$ which is centered in any point of $\mathbb{Z}^{n}$ (not necessarily in $\left.E_{n}\right) . E_{n}$-balls may have different symmetry characteristics depending on where they are centered. Some $E_{n}$-balls are illustrated in Figure 4 for $\mathbb{Z}^{2}$.

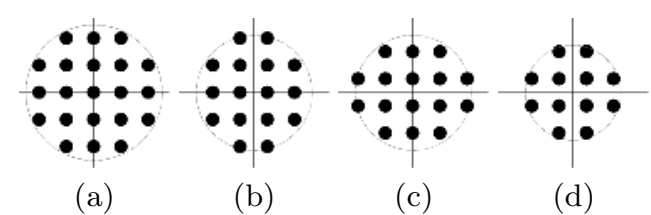

Fig. 4. $E_{n}$-balls in $\mathbb{Z}^{2}$. Only points of $E_{2}$ are represented. (a) $E_{n}$-ball centered in $E_{2}$, (b) and (c) $E_{n}$-balls centered in $E_{1}$, (d) $E_{n}$-ball centered in $E_{0}$.

Definition 10 ( $E_{n}$-maximal balls). Let $\mathrm{X} \subset \mathbb{Z}^{n}, x \in \mathrm{X}, R \in \mathbb{N}$, a) An $E_{n}$-ball $E_{n}\left(B^{<}(x, R)\right) \subseteq \mathrm{X}$ is an $E_{n}$-maximal ball for $x$ in $\mathrm{X}$ if it is the largest $E_{n}$-ball centered in $x$ and included in $\mathrm{X}$, i.e., $\forall R^{\prime} \in \mathbb{N}, E_{n}\left(B^{<}(x, R)\right) \subseteq$ $E_{n}\left(B^{<}\left(x, R^{\prime}\right)\right) \subseteq \mathrm{X} \Rightarrow E_{n}\left(B^{<}(x, R)\right)=E_{n}\left(B^{<}\left(x, R^{\prime}\right)\right)$;

b) An $E_{n}$-ball $E_{n}\left(B^{<}(x, R)\right) \subseteq \mathrm{X}$ is an $E_{n}$-maximal ball for the set $\mathrm{X}$ if it is not strictly included in any other $E_{n}$-ball included in $\mathrm{X}$, i.e., $\forall R^{\prime} \in \mathbb{N}, \forall y \in$ $\mathbb{Z}^{n}, E_{n}\left(B^{<}(x, R)\right) \subseteq E_{n}\left(B^{<}\left(y, R^{\prime}\right)\right) \subseteq \mathrm{X} \Rightarrow E_{n}\left(B^{<}(x, R)\right)=E_{n}\left(B^{<}\left(y, R^{\prime}\right)\right)$.

Definition 11 (HMA). Let $\mathrm{X} \subset \mathbb{Z}^{n}$. The higher resolution medial axis $H M A(\mathrm{X})$ is the set of centers of all $E_{n}$-maximal balls of $\mathcal{H}(\mathrm{X})$.

The problem of extracting the HMA is to find the set of $E_{n}$-maximal balls for the object. Knowing the radii of the $E_{n}$-maximal balls for each point is necessary to test maximality for the object. Let $\mathrm{X} \subset \mathbb{Z}^{n}, \mathrm{X}_{h}=\mathcal{H}(\mathrm{X}), x \in \mathrm{X}_{h}$, in $n \mathrm{D}$ the radius of the $E_{n}$-maximal ball for $x$ can be obtained from $D_{\mathrm{X}_{h}}^{2}(x)$ by looking for the first integer $R \geq D_{\mathrm{X}_{h}}^{2}(x)$ such that $x+v \in E_{n}\left(\overline{\mathrm{X}_{h}}\right)$, with $v$ being any vector satisfying $v^{2}=R$. However, in $2 \mathrm{D}$ and $3 \mathrm{D}$, we can avoid such iterations, 
based on the following proposition, which ensures that $D_{\mathrm{X}_{h}}^{2}(x)$ is precisely the radius of the $E_{n}$-maximal ball for $x$ in $\mathrm{X}_{h}$. It can be proved with the help of Proposition 3 .

Proposition 4. Let $n \in \mathbb{N}, n \leq 3$. Let $\mathrm{X} \subset \mathbb{Z}^{n}, \mathrm{X}_{h}=\mathcal{H}(\mathrm{X}), x \in \mathrm{X}_{h}, R \in \mathbb{D}_{n}$. If $B^{<}(x, R)$ is maximal for $x$ in $\mathrm{X}_{h}$, then $E_{n}\left(B^{<}(x, R)\right)$ is $E_{n}$-maximal for $x$ in $\mathrm{X}_{h}$.

\section{Algorithm to Compute the HMA}

In this section we present an algorithm to compute the higher resolution medial axis (HMA). Testing if an $E_{n}$-ball is maximal for the object is not trivial. One way of doing this is to test if it is not included in another $E_{n}$-ball. This can be done by an adaptation of the algorithm presented by Rémy and Thiel [18 for the extraction of the exact Euclidean medial axis (MA).

Euclidean balls have a number of symmetries that simplify the problem. An Euclidean ball can be reconstructed from only one of its cones (octant in 2D) by retrieving symmetries of each point (or vector) of that cone. We chose the generator cone to be the set of vectors $v^{g} \in \mathbb{Z}^{n}$ such that $v^{g}=\left(v_{1}, v_{2}, \ldots, v_{n}\right), v_{1} \geq$ $v_{2} \geq \cdots \geq v_{n} \geq 0$. We distinguish two types of symmetries of $v^{g}$ :

Type 1. A symmetry obtained by setting signs to the coordinates of $v^{g}$. It can be obtained by $S_{1} v^{g}$, where $S_{1}$ is a matrix in which every element in the diagonal equals 1 or -1 , with 0 s everywhere else. A vector in $\mathbb{Z}^{n}$ has therefore $2^{n}$ Type 1 symmetries, which gives 4,8 and 16 for $n=2,3$ and 4 respectively. We denote by $S_{1}^{n}$ the set of all Type 1 matrices in $n \mathrm{D}$.

Type 2. A symmetry obtained by a permutation of the coordinates of $v^{g}$. It can be obtained by $S_{2} v^{g}$, where $S_{2}$ is a matrix obtained by permuting the rows of an identity matrix according to some permutation of the numbers 1 to $n$. Every row and column therefore contains precisely a single 1 with 0 s everywhere else. A vector in $\mathbb{Z}^{n}$ has therefore $n$ ! Type 2 symmetries, which gives 2,6 and 24 for $n=2,3$ and 4 respectively. We denote by $S_{2}^{n}$ the set of all Type 2 matrices in $n \mathrm{D}$.

For the algorithm we are going to present in this section, we need all the combinations of symmetries of Types 1 and 2 . Any symmetry of $v^{g}$ in $\mathbb{Z}^{n}$ can be obtained by $S_{1} S_{2} v^{g}, S_{1} \in S_{1}^{n}, S_{2} \in S_{2}^{n}$. There are therefore $2^{n} n$ ! symmetries in $\mathbb{Z}^{n}$, which gives 8,48 and 384 for $n=2,3$ and 4 respectively. We denote by $S^{n}$ the set of all products $S_{1} S_{2}$, with $S_{1} \in S_{1}^{n}$, and $S_{2} \in S_{2}^{n}$.

\subsection{Algorithm for the Medial Axis (Rémy and Thiel)}

Given a set $\mathrm{X} \in \mathbb{Z}^{n}$ and its square Euclidean distance transform $D_{\mathrm{X}}^{2}$, the algorithm proposed by Rémy and Thiel [18] tests, for each point $x \in \mathrm{X}$, if the maximal ball for $x$ in $\mathrm{X}, B^{<}\left(x, D_{\mathrm{X}}^{2}(x)\right)$, is a maximal ball for $\mathrm{X}$. This is done 
by testing if $B^{<}\left(x, D_{\mathrm{X}}^{2}(x)\right)$ is not included in another ball in $\mathrm{X}$. If the maximal radius of all maximal balls for $\mathrm{X}$ is not greater than a previously known radius $R_{\text {max }} \in \mathbb{N}$, the inclusion test can be performed efficiently with the help of previously computed lookup tables. These lookup tables are described below and the algorithms to compute them shall be found in references [18, 19.

- Let $\mathrm{X} \in \mathbb{Z}^{n}, x \in \mathrm{X}$, if the maximal radius of all maximal balls for the set $\mathrm{X}$ is not greater than a previously known radius $R_{\max } \in \mathbb{N}$, it is possible to precompute a limited set of generator vectors $\mathcal{M}_{R_{\max }}^{g}$ which is sufficient to ensure that, if $\forall v^{g} \in \mathcal{M}_{R_{\max }}^{g}, \forall S \in S^{n}, B^{<}\left(x, D_{\mathrm{X}}^{2}(x)\right) \not \subset B^{<}\left(x+S v^{g}, D_{\mathrm{X}}^{2}(x+\right.$ $\left.S v^{g}\right)$ ), then $x \in \mathrm{MA}(\mathrm{X}) . \mathcal{M}_{R_{\max }}^{g}$ is the set of sufficient vectors for the radius $R_{\max }$.

- Let $x \in \mathbb{Z}^{n}, v^{g} \in \mathcal{M}_{R_{\max }}^{g}$, for any value of $R_{\max }$, let $R \in \mathbb{D}_{n}$. The table Lut $\left[v^{g}, R\right]$ gives the minimal radius $R^{\prime} \in \mathbb{D}_{n}$ necessary for having $\forall S \in$ $S^{n}, B^{<}(x, R) \subseteq B^{<}\left(x+S v^{g}, R^{\prime}\right)$. Note that $L u t[0, R]=R$.

To compute the MA of a set $\mathrm{X} \in \mathbb{Z}^{n}$, it is sufficient to apply the IsMA function for every point $x \in \mathrm{X}$. The correctness of Function IsMA lies on Proposition 5 and Proposition 6, proved in 18 .

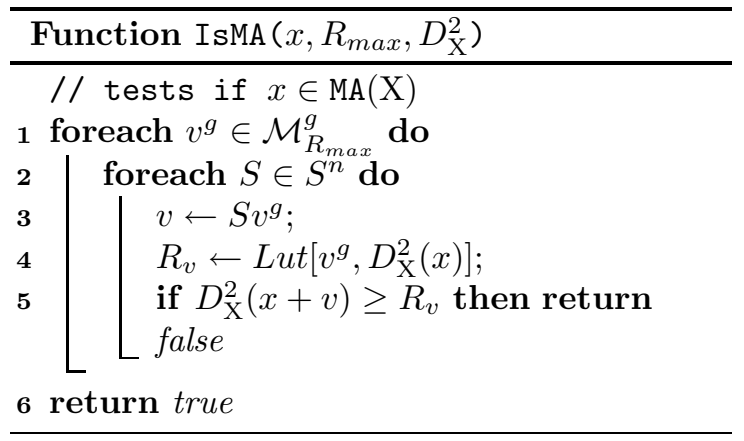

Proposition 5. Let $x \in \mathbb{Z}^{n}, v^{g} \in \mathcal{M}^{g}, S \in S^{n}, v=S v^{g}, R \in \mathbb{D}_{n}, R^{\prime} \in \mathbb{D}_{n}$, we have $B^{<}(x, R) \subseteq B^{<}\left(x+v, R^{\prime}\right) \Leftrightarrow R^{\prime} \geq \operatorname{Lut}\left[v^{g}, R\right]$.

Proposition 6. Let $\mathrm{X} \subset \mathbb{Z}^{n}, x \in \mathrm{X}, R_{\max }=\max \left\{D_{\mathrm{X}}^{2}(z) \mid z \in \mathrm{X}\right\}$. The ball $B^{<}\left(x, D_{\mathrm{X}}^{2}(x)\right)$ is maximal for $\mathrm{X}$ if and only if $\forall v^{g} \in \mathcal{M}_{R_{\max }}^{g}, \forall S \in$ $S^{n}, B^{<}\left(x, D_{\mathrm{X}}^{2}(x)\right) \not \subset B^{<}\left(x+v, D_{\mathrm{X}}^{2}(x+v)\right)$ where $v=S v^{g}$.

\subsection{Algorithm to Extract the HMA}

The MA algorithm presented above takes profit of the minimal radii given by the lookup table $L u t$. We need a notion of minimal radius for the $E_{n}$-balls.

Definition 12 ( $E_{n}$-minimal radius). Let $x \in \mathbb{Z}^{n}, R \in \mathbb{D}_{n}, v \in \mathbb{Z}^{n}$, the $E_{n^{-}}$ minimal radius relative to $x, R$ and $v$, denoted by $R^{\vee}(x, R, v)$, is the strict radius of the smallest ball centered in $x+v$ which includes $E_{n}\left(B^{<}(x, R)\right)$, i.e., $R^{\vee}(x, R, v)=\min \left\{R^{\prime} \in \mathbb{D}_{n}, E_{n}\left(B^{<}(x, R)\right) \subseteq E_{n}\left(B^{<}\left(x+v, R^{\prime}\right)\right)\right\}$. 
The following proposition is elementary.

Proposition 7. Let $x \in \mathbb{Z}^{n}, R \in \mathbb{D}_{n}, R^{\prime} \in \mathbb{D}_{n}, v \in \mathbb{Z}^{n}, R^{\prime}<R^{\vee}(x, R, v) \Leftrightarrow$ $E_{n}\left(B^{<}(x, R)\right) \nsubseteq E_{n}\left(B^{<}\left(x+v, R^{\prime}\right)\right)$.

Unlike Euclidean balls in $\mathbb{Z}^{n}, E_{n}$-balls may not be invariant by symmetries of Type 2. Thus the value of $R^{\vee}(x, R, v)$ depends on to which subset $E_{i}$ of $\mathbb{Z}^{n}$ belong the points $x$ and $x+v$. The construction of a lookup table with $E_{n^{-}}$ minimal radii may be prohibitive. We propose to calculate $R^{\vee}(x, R, v)$ on runtime with the Function EnRmin.

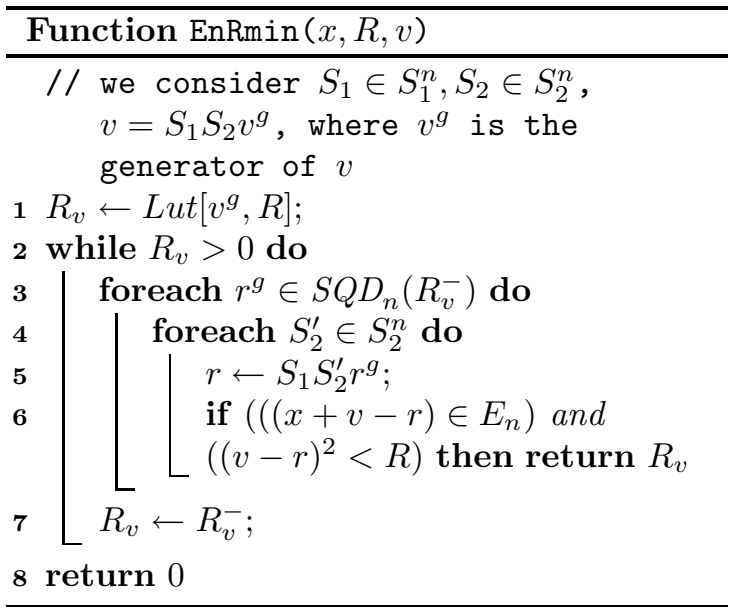

Proposition 8. The value $R_{v}$ returned by the EnRmin function is equal to $R^{\vee}(x, R, v)$.

An example with Function EnRmin is given in [19] and may be useful for its comprehension.

Now we need to construct a table $\mathcal{M}^{h}$, which gives the set of vectors sufficient to compute the HMA. The construction of this table is done by the BuildMhLut procedure presented in [19. This procedure, similar to the one of 18 to compute $\mathcal{M}^{g}$, is based on the observation that if $\mathcal{M}_{R_{\text {max }}}^{h}$ is sufficient to extract, from any ball with a radius less or equal to $R_{\max }$, a medial axis which is reduced to a single point, then $\mathcal{M}_{R_{\max }}^{h}$ enables to extract correctly the HMA from any squared distance map which values do not exceed $R_{\max }$.

By construction of $\mathcal{M}^{h}$ and as a consequence of Proposition 4, Proposition 7 and Proposition 8, we have the two following propositions.

Proposition 9. Let $n \in \mathbb{N}, x \in \mathbb{Z}^{n}, v^{g} \in \mathcal{M}^{h}, S \in S^{n}, v=S v^{g}, R \in \mathbb{N}, R^{\prime} \in \mathbb{N}$. We have $E_{n}\left(B^{<}(x, R)\right) \subseteq E_{n}\left(B^{<}\left(x+v, R^{\prime}\right)\right) \Leftrightarrow R^{\prime} \geq \operatorname{EnRmin}(x, R, v)$.

Proposition 10. Let $n \in \mathbb{N}, n \leq 3$. Let $\mathrm{X} \subset \mathbb{Z}^{n}, \mathrm{X}_{h}=\mathcal{H}(\mathrm{X}), x \in \mathrm{X}_{h}, R_{\max }=$ $\max \left\{D_{\mathrm{X}_{h}}^{2}\left(x^{\prime}\right), x^{\prime} \in \mathrm{X}_{h}\right\}$. The $E_{n}$-ball $E_{n}\left(B^{<}\left(x, D_{\mathrm{X}_{h}}^{2}(x)\right)\right)$ is $E_{n}$-maximal for $\mathrm{X}_{h}$ if and only if $\forall v^{g} \in \mathcal{M}_{R_{\text {max }}}^{h}, \forall S \in S^{n}, E_{n}\left(B^{<}\left(x, D_{\mathrm{X}_{h}}^{2}(x)\right)\right) \not \subset E_{n}\left(B^{<}(x+\right.$ $\left.\left.v, D_{\mathrm{X}_{h}}^{2}(x+v)\right)\right)$, where $v=S v^{g}$. 
We conclude from Proposition 9 and Proposition [10 that, given a set $\mathrm{X} \subset$ $\mathbb{Z}^{n}$, the computation of $\operatorname{HMA}(\mathrm{X})$ may be done by the computation of the IsHMA function for every point $x \in \mathcal{H}(\mathrm{X})$. Note the direct use of $D_{\mathrm{X}_{h}}^{2}$, valid only in $2 \mathrm{D}$ and $3 \mathrm{D}$, thanks to Proposition 4 .

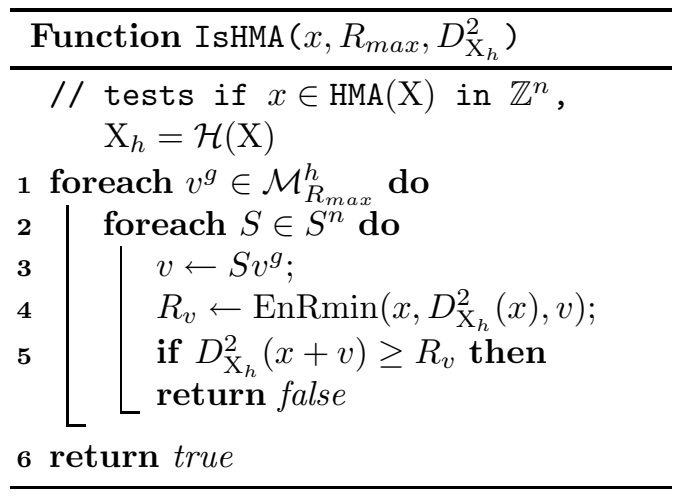

To efficiently calculate $R^{+}, R^{-}$for any integer $R$, another precomputed lookup table is used: the square decompositions table $\mathrm{SQD}_{n}$ defined in Section 2 . The computation of such table is very simple in any dimension, but the algorithm is available in 19,4 .

Let $R \in \mathbb{N}, x \in \mathbb{Z}^{n}$, calculations are expressed as follows:

$$
\begin{aligned}
& \bar{R}:\left(\text { while }\left(\mathrm{SQD}_{n}(R)=\emptyset\right) \text { do } R \leftarrow R+1\right) ; \quad R^{+}:(R \leftarrow R+1 ; R \leftarrow \bar{R}) \\
& \underline{R}:\left(\text { while }\left(\operatorname{SQD}_{n}(R)=\emptyset\right) \text { do } R \leftarrow R-1\right) ; \quad R^{-}:(R \leftarrow R-1 ; R \leftarrow \underline{R})
\end{aligned}
$$

\section{Results}

Besides the didactic example given in Figure 1(a), in Figure 5 we present a practical 2D example of the HMA, comparing it to the MA.
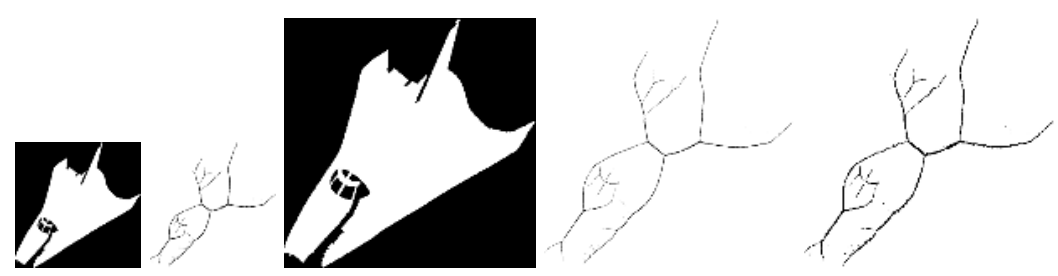

Fig. 5. 2D HMA example. From left to right: the original object, its MA, the object in doubled resolution, its HMA and, for comparison, the MA scaled to a double resolution.

As a practical evaluation of the HMA computation time, we have executed MA and HMA on several images similar to those presented in Figure 5 and also on 3D segmentations of a cerebral structure called hippocampus. The estimations were performed on an AMD Athlon XP 2400+, 2.0 GHz, running Linux, without compiler optimizations.

Let $\mathrm{X}$ be the object. We measured the times $t(\mathrm{X})$ and $t_{h}(\mathrm{X})$ for computing $\mathrm{MA}(\mathrm{X})$ and $\operatorname{HMA}(\mathrm{X})$, respectively. 
For the 2D images, we have zoomed the images by factors of $0.5,1$ and 2 in order to have different ball sizes in similar shapes. The object sizes are 12445,50157 and 112842 pixels. We obtained for $t(\mathrm{X}): 0.187,0.426,0.662$ and for $t_{h}(\mathrm{X}): 0.487,1.281,2.906$ (in seconds). The ratios between $t_{h}(\mathrm{X})$ and $t(\mathrm{X})$ are respectively 2.6,3.0 and 4.4. The fact that we obtained ratios below four, whereas doubling the resolution multiplies the data volume by four in $2 \mathrm{D}$, is mainly due to the relative cost of loading the lookup tables: this cost is significant for small images but is better amortized for large images. Notice, however, that only the parts of the lookup tables which are really needed are loaded.

For the 3D images, we used zooming factors of 1,1.5 and 2, and the object sizes are 3530,11799 and 24771 voxels. We obtained for $t(\mathrm{X}): 0.077,0.148,0.330$, and for $t_{h}(\mathrm{X}): 0.422,2.01,5.36$; hence the ratios: $5.45,13.5,16.2$. Notice that in $3 \mathrm{D}$, doubling the resolution multiplies the data volume by eight.

We also evaluated and compared the "thinness" of the HMA and the one of the MA. Let us denote by $T(\mathrm{X})$ the ratio: $\operatorname{size}(\mathrm{MA}(\mathrm{X})) / \operatorname{size}(\mathrm{X})$, and by $T_{h}(\mathrm{X})$ the ratio: $\operatorname{size}(\mathrm{HMA}(\mathrm{X})) / \operatorname{size}(\mathcal{H}(\mathrm{X}))$. In $2 \mathrm{D}$, we obtained for the three zoomed images: $T(\mathrm{X})=0.091,0.054,0.042$, and $T_{h}(\mathrm{X})=0.035,0.022,0.017$ respectively. In $3 \mathrm{D}$, we obtained for the three zoomed images: $T(\mathrm{X})=0.33,0.26,0.24$, and $T_{h}(\mathrm{X})=0.067,0.072,0.077$ respectively.

It was interesting to note in the $2 \mathrm{D}$ case that the bigger is $\mathrm{X}$, the thinner is $\mathrm{MA}(\mathrm{X})$, and that the HMA offers better improvements for small objects. In 3D, the same observation may be done, and the improvements brought by the HMA are more sensible than in $2 \mathrm{D}$.

\section{Conclusion and Perspectives}

We have defined the HMA - the exact Euclidean medial axis in higher resolution in a completely discrete framework. We showed that the HMA presents better thinness characteristics than the classical discrete Euclidean MA. We explained how to compute such axis in $n$-dimensions and we provided an efficient algorithm to compute it in $2 \mathrm{D}$ and $3 \mathrm{D}$. Although the proofs could not be presented here, the algorithm has been systematically proved. We presented a practical evaluation of the algorithm's behaviour in terms of speed. A more precise complexity analysis will be given in the extended version of this paper. The HMA is based on a transformation to higher resolution which permits the application of further homotopic thinning for the computation of homotopic skeletons. In a forthcoming paper 22, we define the notion of a symmetric homotopic skeleton which contains the Euclidean HMA, and provide a parallel algorithm to compute it, based on the framework of critical kernels (see [10]). We also intend to exploit a different approach (see [17]) in a future work, in order to obtain an even more efficient algorithm, generalized to $n$ dimensions.

\section{References}

1. Blum, H.: An associative machine for dealing with the visual field and some of its biological implications. Biological prototypes and synthetic systems 1 (1961) 244-260 
2. Davies, E., Plummer, A.: Thinning algorithms: a critique and a new methodology. Pattern Recognition 14 (1981) 53-63

3. Talbot, H., Vincent, L.: Euclidean skeletons and conditional bisectors. In: Procs. VCIP'92, SPIE. Volume 1818. (1992) 862-876

4. Couprie, M., Coeurjolly, D., Zrour, R.: Discrete bisector function and euclidean skeleton in 2d and 3d. Image and Vision Computing (2006) accepted.

5. Bertrand, G.: Skeletons in derived grids. In: procs. Int. Conf. Patt. Recogn. (1984) 326-329

6. Kovalevsky, V.: Finite topology as applied to image analysis. Computer Vision, Graphics and Image Processing 46 (1989) 141-161

7. Khalimsky, E., Kopperman, R., Meyer, P.: Computer graphics and connected topologies on finite ordered sets. Topology and its Applications 36 (1990) 1-17

8. Kong, T.Y., Kopperman, R., Meyer, P.: A topological approach to digital topology. American Mathematical Monthly 38 (1991) 901-917

9. Bertrand, G.: New notions for discrete topology. In: procs. DGCI, LNCS, Springer Verlag. Volume 1568. (1999) 216-226

10. Bertrand, G., Couprie, M.: New 3d parallel thinning algorithms based on critical kernels. In Kuba, A., Palágyi, K., Nyúl, L., eds.: DGCI. LNCS, Springer (2006)

11. Danielsson, P.: Euclidean distance mapping. Computer Graphics and Image Processing 14 (1980) 227-248

12. Meyer, F.: Cytologie quantitative et morphologie mathématique. PhD thesis, École des Mines de Paris, France (1979)

13. Saito, T., Toriwaki, J.: New algorithms for euclidean distance transformation of an n-dimensional digitized picture with applications. Pattern Recognition 27 (1994) $1551-1565$

14. Hirata, T.: A unified linear-time algorithm for computing distance maps. Information Processing Letters 58(3) (1996) 129-133

15. Meijster, A., Roerdink, J., Hesselink, W.: A general algorithm for computing distance transforms in linear time. In J. Goutsias, L.V., Bloomberg, D., eds.: Mathematical morphology and its applications to image and signal processing 5 th. Volume 18 of Computational Imaging and Vision., Kluwer Academic Publishers (2000) 331-340

16. Rémy, E., Thiel, E.: Look-up tables for medial axis on squared Euclidean distance transform. In: procs. DGCI, LNCS, Springer Verlag. Volume 2886. (2003) 224-235

17. Cœurjolly, D.: d-dimensional reverse Euclidean distance transformation and Euclidean medial axis extraction in optimal time. In: procs. DGCI, LNCS, Springer Verlag. Volume 2886. (2003) 327-337

18. Rémy, E., Thiel, E.: Exact medial axis with euclidean distance. Image and Vision Computing 23(2) (2005) 167-175

19. Saúde, A.V., Couprie, M., Lotufo, R.: Exact Euclidean medial axis in higher resolution. Technical Report IGM2006-5, IGM, Université de Marne-la-Vallée (2006)

20. Borgefors, G., Ragnemalm, I., di Baja, G.S.: The Euclidean Distance Transform: finding the local maxima and reconstructing the shape. In: Seventh Scandinavian Conference on Image Analysis. Volume 2., Aalborg, Denmark (1991) 974-981

21. Hardy, G., Wright, E.: An Introduction to the Theory of Numbers. 5th edn. Oxford University Press (1978)

22. Couprie, M., Saúde, A.V., Bertrand, G.: Euclidean homotopic skeleton based on critical kernels. In: Procs. SIBGRAPI. (2006) to appear. 